\title{
DIFERENCIAS ESPACIO-TEMPORALES DE LA LOCOMOCIÓN EN ADULTOS VARONES CON NORMOPESO Y SOBREPESO
}

\author{
DIFERENÇAS ESPAÇO-TEMPORAIS NA LOCOMOÇÃO DEADULTOS DO SEXO MASCULINO COM PESO \\ NORMAL ESOBREPESO
}

Artículo Original

Artigo ORIGINAL

ORIGINAL ARTICLE

\author{
SPATIO-TEMPORAL DIFFERENCES OF LOCOMOTION OF ADULT MALES WITH NORMAL WEIGHTAND \\ OVERWEIGHT
}

\begin{abstract}
José Maria Heredia-Jiménez 1 (Profissional de Educação Física)

Sara Mallagaray-Corral ${ }^{1}$ (Profissional de Educação Física) Eva Orantes-González (Profissional de Educação Física) Víctor Manuel Soto-Hermoso (Profissional de Educação Física)
\end{abstract}

1. Universidad de Granada, Departamento de Educación Física y Deportiva, Granada, España.

\section{Correspondência:}

Departamento Educación Física y Deportiva. Universidad de Granada. Crta de Alfacar s/n. 18071, Granada, España. herediaj@ugr.es

\section{RESUMEN}

Introducción: La obesidad ha estado relacionada con la presencia de adaptaciones estructurales y funcionales que provocan limitaciones en el control del movimiento. Objetivo: Determinar el efecto del exceso de peso sobre las variables espacio-temporales de la locomoción en varones jóvenes y activos. Métodos: Estudio de corte transversal de muestreo no probabilístico con 55 sujetos con normopeso (IMC $\leq 25$ ) y 22 sujetos con sobrepeso (IMC >25). Se analizó el IMC, \% de masa grasa, y el \% de masa magra de ambos grupos con un bioimpedanciómetro multifrecuencia (Inbody 230). También se analizaron las variables cinemáticas de la locomoción utilizando una plataforma de presiones GaitRite. Resultados: El grupo con sobrepeso mostró una disminución significativa de la fase de oscilación y fase de apoyo monopodal $(p<0,001)$ junto con un aumento de la fase de apoyo y fase de apoyo bipodal $(p<0,001)$ comparado con el grupo normopeso. Conclusión: La reducción de la fase de oscilación y el incremento de la fase de apoyo y fase de apoyo bipodal que manifestaron el grupo con sobrepeso puede ser consecuencia del aumento de la inestabilidad que se produce durante la marcha debido al sobrepeso.

Descriptores: fenómenos biomecánicos; locomoción; obesidad; análisis espacio-temporal.

\section{RESUMO}

Introdução: A obesidade tem sido relacionada com a presença de adaptações estruturais e funcionais que podem limitar o controle dos movimentos. Objetivo: Determinar o efeito do excesso de peso sobre as variáveis espaço-temporais da locomoção em homens jovens e ativos. Métodos: Estudo transversal de amostragem não probabilística, com 55 indivíduos com peso normal (IMC $\leq 25$ ) e 22 indivíduos com sobrepeso (IMC > 25). Analisaram-se IMC e porcentagem de massa gorda e massa magra em ambos os grupos, com um bioimpedanciômetro multifrequência (Inbody 230). Foram analisadas também as variáveis cinemáticas de locomoção, usando-se um sistema de plataforma de pressão (GaitRite). Resultados: O grupo sobrepeso apresentou diminuição significativa na fase de balanço e fase de apoio monopodal $(p<0,001)$, com aumento da fase de apoio e da fase de apoio bipodal ( $p<0,001)$ com relação ao grupo peso normal. Conclusão: A redução da fase de balanço e o aumento da fase de apoio e da fase de apoio bipodal manifestado pelo grupo sobrepeso pode ser resultado do aumento da instabilidade que ocorre durante a marcha, devido ao sobrepeso.

Descritores: fenômenos biomecânicos; locomoção; obesidade; análise espaço-temporal.

\begin{abstract}
Introduction: Obesity has been associated with the presence of structural and functional adaptations that may limit movement control. Objective: To determine the effect of overweight on spatio-temporal variables of locomotion in young and active men. Methods: A cross-sectional study of non-probability sampling, with 55 subjects with normal weight (BMI $\leq 25)$ and 22 overweight subjects (BMI >25). Body mass index and percentage of fat mass and lean mass were analyzed in both groups, with a multi-frequency bioimpedanciometer (Inbody 230). The kinematic variables of locomotion were also analyzed with a pressure platform system (GaitRite). Results: The overweight group showed a significant decrease in the swing phase and monopodal stance phase $(p<0.001)$ with an increase in the stance phase and double stance phase $(p<0.001)$ in relation to the normal weight group. Conclusion: The reduction of the swing phase and the increase of the stance phase and double stance phase presented by the overweight group may be a result of increased instability that occurs during gait due to overweight.
\end{abstract}

Keywords: biomechanical phenomena; locomotion; obesity; spatio-temporal analysis.

\section{INTRODUCCIÓN}

La marcha humana es un proceso que implica una compleja interacción entre las fuerzas musculares y las fuerzas inerciales que resultan de la adaptación progresiva del cuerpo a través del espacio y de reducir al mínimo el gasto de energía'. La importancia de los modelos de marcha humana obtenidos a partir de investigaciones sobre 
biomecánica de la locomoción, radica en el hecho de que su análisis puede servir de gran ayuda para detectar diversos trastornos clínicos², para evaluar la calidad de vida relacionada con la salud ${ }^{3,4}$, para predecir el riesgo de sufrir caídas ${ }^{5}$ e incluso el riesgo de mortalidad temprana ${ }^{6}$. Mundialmente la obesidad está aumentando en la última década ${ }^{7}$. En España la prevalencia de sobrepeso es del 34,2\%, siendo mayor en los varones (43,9\%) que en las mujeres (25,7\%); y la de obesidad del 13,6\%, sin existir diferencias significativas entre $\operatorname{sexos}^{8}$.

La obesidad se asocia a menudo con limitaciones estructurales y funcionales que pueden restringir el control del movimiento 9 e influir en los patrones de la locomoción. Es por ello, que diversos estudios se centran en analizar las variables espacio-temporales de la locomoción en individuos obesos, extrayendo que la obesidad se asocia con una disminución significativa de la velocidad $(V)$ y de la longitud de zancada $(L Z)^{10}$, y con una fase de apoyo (FA) y fase de apoyo bipodal (FAB) significativamente mayores en obesos ${ }^{10,11}$. Además, la fase de oscilación (FO) es menor ${ }^{11}$ y la anchura de paso mayor ${ }^{12}$ en personas obesas, estando éstos cambios directamente asociados al mantenimiento del equilibrio dinámico ${ }^{13}$. Además estos cambios en la locomoción implican una mayor inestabilidad en comparación con los que poseen un peso normal ${ }^{10}$.

Existe un número limitado de estudios que han investigado las limitaciones del exceso de peso corporal en la locomoción humana. Algunos hacen referencia a las características espacio-temporales de la locomoción en adultos obesos ${ }^{12}$, otros a la influencia de la obesidad en el decremento de la fuerza muscular y la potencia ${ }^{14-16}$ o en la menor eficiencia energética durante la locomoción ${ }^{17-19}$. Son pocos los estudios que analizan las características espacio-temporales de la locomoción en sujetos con diferentes índices de masa corporal (IMC), bien en mujeres ${ }^{20}$ o en mayores ${ }^{21}$ y sólo dos estudios que consideren la masa grasa de las mujeres además del IMC ${ }^{13,15}$. Además cabe destacar que ninguno de estos estudios se ha llevado a cabo con población española.

Existe cierta controversia sobre los resultados de estudios previos, así Spyropoulos et al. ${ }^{12}$ y Lai et al. ${ }^{10}$ mostraron que tanto la $V$ como la LZ presentaban una disminución significativa en poblaciones obesas en comparación con personas normopeso. Por otro lado, estudios como los de Blaszczyk et al. ${ }^{20}$ y Wu et al. ${ }^{13}$ argumentan que no existen cambios significativos en dichas variables. Sin embargo, en la investigación llevada a cabo por Ko et al. ${ }^{21}$ en mayores, al comparar individuos con normopeso, sobrepeso y obesidad, se observan resultados diferentes en variables como la $V$ y la FA, encontrándose diferencias significativas entre el grupo con normopeso y el de obesos, pero no significativas si se compara el grupo de normopeso contra el de sobrepeso.

Debido a dicha controversia y al limitado número de estudios que analicen desde un enfoque cinemático la locomoción en población adulta joven con sobrepeso, el objetivo de este estudio es determinar los efectos del exceso de peso corporal sobre las características espacio-temporales de la locomoción en varones con normopeso y sobrepeso.

\section{MATERIAL Y METODO}

Setente y siete hombres adultos participaron en el presente estudio (Tabla 1). Se dividió la muestra en dos grupos tomando como referencia valores de IMC de acuerdo con los criterios de la OMS 22 : un grupo normopeso que estaba formado por 55 sujetos cuyo IMC $\leq 25 \mathrm{~kg} / \mathrm{m}^{2}$ y otro grupo sobrepeso el cual estaba formado por 22 sujetos con un IMC $>25 \mathrm{~kg} / \mathrm{m}^{2}$.

Todos los sujetos participaron de forma voluntaria en el estudio. Se les informó por escrito de los objetivos, beneficios y posibles consecuencias del mismo así como la posibilidad de aclarar cualquier duda o pregunta que les fuera surgiendo durante la realización del estudio. A continuación firmaron una hoja de consentimiento informado. El estudio ha sido aprobado por el Comité Ético de la Universidad de Granada (núm:201). Todos los sujetos estaban aparentemente sanos y ninguno de ellos padecía enfermedades musculoesqueléticas o neurológicas que pudieran alterar la locomoción y provocar modificaciones en los resultados del estudio. Además, todos los sujetos eran físicamente activos ya que realizaban al menos dos sesiones por semana ${ }^{23}$.

\section{MATERIAL Y PROCEDIMIENTO}

Los sujetos fueron tallados descalzos con un estadiómetro (Harpenden, Byomedic System, España) siendo la unidad de medida de 0,1 cm y una precisión de $1 \mathrm{~mm}$ y posteriormente se analizó el peso y la composición corporal. Ambas medidas fueron tomadas con un bioimpedanciometro multifrecuencia de 8 electrodos (Inbody 230, Biospace, Korea). Para realizar dicha prueba, 24 horas antes los sujetos no realizaron ejercicio físico ni se sometieron a una sauna. No ingirieron ningún alimento al menos las $4 \mathrm{~h}$ previas a la prueba, ni se aplicaron cremas o aceites corporales. Se les liberó de la mayor cantidad de ropa posible y accesorios metálicos (pendientes, pulseras, etc.) y permanecieron al menos 5 minutos de pie antes de la prueba ${ }^{24,25}$.

Para la realización de la prueba de locomoción se utilizó una plataforma de presiones que permite la evaluación de los parámetros espacio-temporales (GAITRite system; CIR Systems Inc, Clifton, NJ, EE.UU). El sistema GAITRite estaba formado por una alfombra de marcha de $4,6 \mathrm{~m}$ de longitud con un total de 16128 sensores. El área activa de los sensores es de $0,61 \mathrm{~m}$ de anchura por 3,66m de longitud y la frecuencia de muestreo de $80 \mathrm{~Hz}$.

Para la prueba de locomoción se creó un pasillo de marcha de 18,6 m delimitado por un cono en cada extremo, que servirá de zona de giro (Figura 1). La alfombra GAITRite se colocó en el centro, desechando así la toma de datos en el inicio y el fin del pasillo, por ser zonas de aceleración y desaceleración de la marcha. Se siguieron las recomendaciones de calzado y medidas propuestas por el European GAlTRite Network Group ${ }^{26}$.

Cada sujeto caminó por el pasillo al menos cinco veces para poder registrar un mínimo de 20 pasos sobre la plataforma ${ }^{27}$. Los sujetos no dispusieron de ningún tipo de ayuda o apoyo durante la prueba. Durante la realización de la prueba, no se les animó a los sujetos ni se les instó a acelerar o desacelerar su marcha. Antes de comenzar la prueba se les dio a los participantes la siguiente indicación: "Debéis de caminar de la forma más natural posible, como lo hacéis normalmente cuando vais a comprar o a casa de algún amigo". Todas las pruebas del presente estudio fueron realizadas por el mismo investigador y en las mismas condiciones.

Tabla 1. Características antropométricas de los sujetos.

\begin{tabular}{c|c|c}
\hline Variables & Normopeso (N= 55) & Sobrepeso (N= 22) \\
\hline Edad (años) & $24,3(4,8)$ & $27,8(7,5)$ \\
\hline Peso $(\mathrm{kg})$ & $69,9(5,7)$ & $83,5(10,5)$ \\
\hline Talla $(\mathrm{m})$ & $1,77(0,54)$ & $1,78(0,73)$ \\
\hline IMC $\left(\mathrm{kg} / \mathrm{m}^{2}\right)$ & $22,4(1,5)$ & $27,2(2,1)$ \\
\hline \% Masa grasa (\% PC) & $12,2(3,7)$ & $20,3(5,9)$ \\
\hline \% Masa magra (\% PC) & $82,9(3,5)$ & $75,1(5,6)$ \\
\hline \%PC: \% peso corporal. & & \\
\hline
\end{tabular}

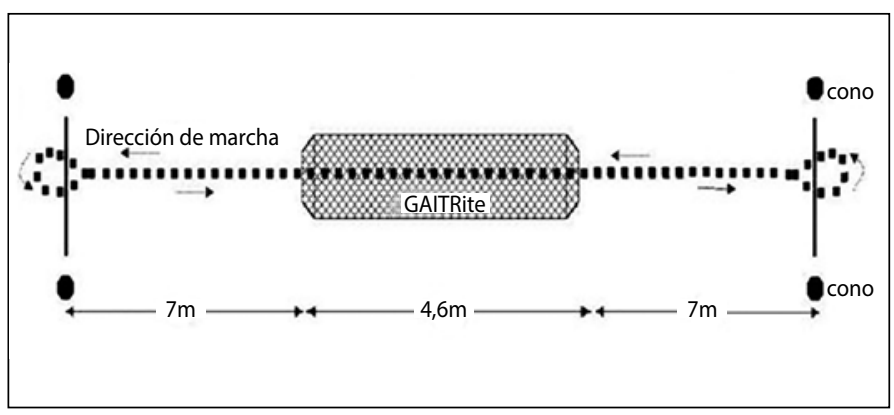

Figura 1. Diseño del pasillo de locomoción del estudio. 


\section{Variables y diseño}

El presente estudio es de corte transversal. Se realizó un muestreo no probabilístico de los sujetos. Como variable de manipulación se seleccionó el IMC de los sujetos, con dos niveles: sujetos normopeso y sujetos con sobrepeso.

Se evaluaron las siguientes variables: peso $(\mathrm{kg})$, talla $(\mathrm{m})$; IMC $\left(\mathrm{kg} / \mathrm{m}^{2}\right)$; porcentaje de masa grasa (\%) y porcentaje de masa magra (\%) y las variables cinemáticas de la locomoción, que se detallan en la Tabla 2. Tanto las variables de locomoción como las variables de composición corporal han sido calculadas según las ecuaciones propuestas por los fabricantes de los equipos.

\section{Análisis estadístico}

Se realizó una estadística descriptiva de las variables analizadas, expresándose todas las variables como media y desviación típica. Se realizó posteriormente la prueba de normalidad de Shapiro-Wilk. La comparación entre grupos se realizó, para las variables paramétricas, mediante el test $t$ Student para muestras independientes, controlando la homocedasticidad de las variables mediante el test de Levene. Las variables que no cumplieron el supuesto de normalidad fueron comparadas mediante el test de Mann-Whitney. El nivel de significación fue de $p<0,05$. Se utilizó el software Statistical Package for the Social Science (SPSS) versión 19.0 (SPSS. Chicago. IL. EE.UU.).

\section{RESULTADOS}

En la Tabla 3 se muestran los datos comparativos de las variables espacio-temporales de la locomoción en los dos grupos estudiados.

En lo que respecta a las variables espacio-temporales de la locomoción, el grupo sobrepeso mostró valores significativamente menores en la FO y FAM y valores significativamente mayores en la FA y la FAB respecto al grupo normopeso (Figura 2). Las variables V, LZ y CAD, no mostraron diferencias significativas entre ambos grupos.

\section{DISCUSIÓN}

El objetivo de esta investigación fue determinar los efectos del exceso de peso corporal sobre las variables espacio-temporales de la locomoción en varones jóvenes activos con normopeso y sobrepeso. Los resultados de este estudio muestran que los jóvenes varones con sobrepeso modifican sus patrones de marcha en comparación con los que poseen normopeso, confirmando que un exceso de peso

Tabla 2. Definición de las variables espacio-temporales de la locomoción que han sido analizadas.

\begin{tabular}{|c|c|c|}
\hline Variables & Definición & Unidad \\
\hline $\begin{array}{c}\text { Velocidad de la } \\
\text { marcha }(V)\end{array}$ & $\begin{array}{c}\text { Distancia cubierta por el cuerpo } \\
\text { entre tiempo transcurrido. }\end{array}$ & $\mathrm{cm} / \mathrm{s}$ \\
\hline Cadencia (CAD) & $\begin{array}{c}\text { Numero de pasos ejecutados } \\
\text { en unidad de tiempo. }\end{array}$ & Pasos/min \\
\hline $\begin{array}{l}\text { Longitud de } \\
\text { Zancada (LZ) }\end{array}$ & $\begin{array}{l}\text { Distancia de la línea de progresión } \\
\text { entre el punto del talón de dos apoyos } \\
\text { consecutivos del mismo pie }\end{array}$ & $\begin{array}{l}\% \text { ciclo de } \\
\text { marcha }\end{array}$ \\
\hline $\begin{array}{c}\text { Fase de } \\
\text { Oscilación (FO) }\end{array}$ & $\begin{array}{c}\text { Es el tiempo que transcurre entre el último } \\
\text { contacto de la pisada actual al primer contacto } \\
\text { de la siguiente pisada del mismo pie }\end{array}$ & $\begin{array}{l}\text { \% ciclo de } \\
\text { marcha }\end{array}$ \\
\hline Fase de Apoyo (FA) & $\begin{array}{l}\text { Tiempo que transcurre entre el primer } \\
\text { contacto y el último contacto de dos } \\
\text { pisadas consecutivas del mismo pie }\end{array}$ & $\begin{array}{l}\% \text { ciclo de } \\
\text { marcha }\end{array}$ \\
\hline $\begin{array}{c}\text { Fase Apoyo } \\
\text { Monopodal (FAM) }\end{array}$ & $\begin{array}{l}\text { Es el tiempo transcurrido entre el último } \\
\text { contacto del pie y el primer contacto de } \\
\text { la pisada siguiente del mismo pie }\end{array}$ & $\begin{array}{l}\% \text { ciclo de } \\
\text { marcha }\end{array}$ \\
\hline
\end{tabular}

Fase de Apoyo Bipodal (FAB) Tiempo transcurrido desde el apoyo del talón de un pie al despegue de los dedos del pie opuesto. $\%$ ciclo de marcha puede dar lugar a cambios significativos en las variables cinemáticas de la locomoción, debido a la disminución en la FO, FAM y el aumento significativo en la FA y FAB $(p<0,001)$ en el grupo con sobrepeso en comparación con el grupo con normopeso.

La importancia de dicha comparación reside en los efectos que supone el exceso de peso corporal en la FO, los cuales son críticos para las principales tareas de control del aparato locomotor, como son la absorción del impacto, el mantenimiento de la estabilidad en el apoyo y la progresión de la locomoción; condicionando éstas la movilidad y el control muscular ejercido sobre el pie, quien a su vez, desempeña el papel de segmento de contacto con el suelo ${ }^{28}$.

Spyropoulos et al. ${ }^{12}$ mostraron los primeros valores de parámetros cuantitativos espacio-temporales de la marcha en 12 hombres obesos, obteniendo una $V$ de 1,09 m/s y una $L Z$ de 1,25 m, concluyendo que los obsesos andan más lento y con pasos más cortos. Posteriormente se han realizado otros estudios que corroboran dicha información. Lai et al. ${ }^{10}$ en su estudio realizado con adultos obesos, observaron una disminución significativa tanto de la $\vee$ en el grupo obeso (normopeso: $127 \pm 0,17 \mathrm{~cm} / \mathrm{s}$; obeso: $112 \pm 0,10 \mathrm{~cm} / \mathrm{s} ; \mathrm{p}<0,01$ ) como de la $L Z$ respecto a la altura del sujeto (normopeso: 0,77 $\pm 0,06 \mathrm{~m}$; obeso: 0,71 $\pm 0,04 \mathrm{~m}$; $\mathrm{p}<0,01$ ). Además Runhaar et al. ${ }^{29}$ tras realizar una revisión sistemática de estudios con individuos obesos afirma lo mismo. En nuestro estudio dichos valores también son menores para los individuos de mayor peso aunque las diferencias obtenidas entre los dos grupos no son significativas. Esto puede deberse a que en nuestro estudio se compara sujetos con sobrepeso pero no con obesidad.

Tabla 3. Comparación entre grupos de las variables de locomoción.

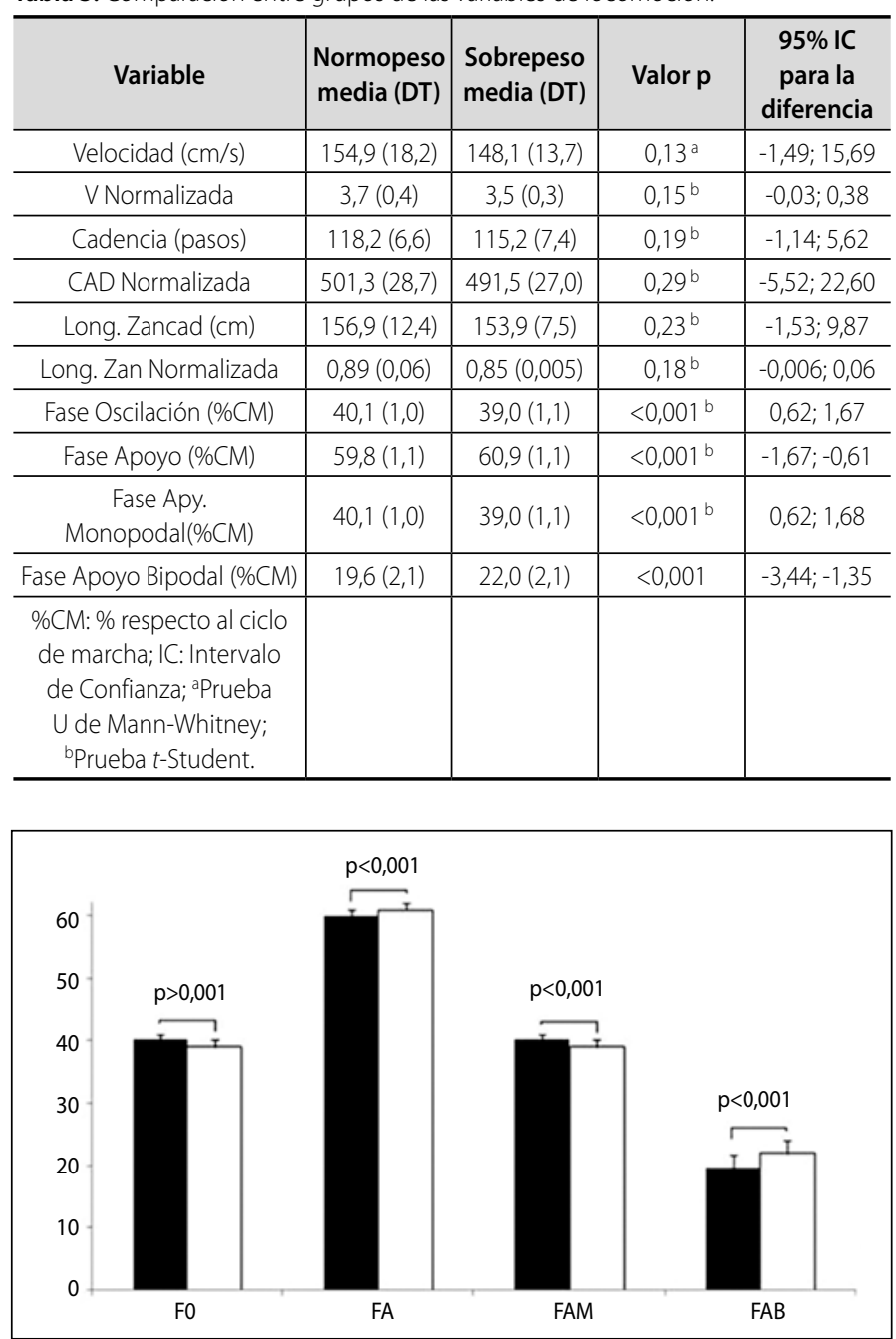

Figura 2. Diferencias en las fases de la locomoción entre grupos. (FO: Fase de oscilación; FA: Fase de Apoyo; FAM: Fase de Apoyo Monopodal; FAB: Fase de Apoyo Bipodal). 
Por otro lado, nuestros hallazgos coinciden con los encontrados por Ko et al. ${ }^{21}$ quienes tampoco obtuvieron diferencias significativas en la V de marcha. Cabe destacar, que en dicho estudio, las $V$ de ambos grupos son inferiores a las observadas por nuestros sujetos, ya que se trata de individuos de mayor edad (67,08 \pm 0,99 años), estando demostrado la reducción de la velocidad con el incremento de los años.

La falta de significación en las variables V, CAD y LZ encontradas en nuestra investigación, concuerdan con los hallazgos de estudios pre$v^{\text {vios }}{ }^{20}$ donde se evaluaron las variables cinemáticas de sujetos obesos y normopeso. Los autores destacaron que los sujetos obesos que caminan con la misma velocidad que los sujetos sanos muestran características similares de locomoción, no encontrando diferencias estadísticas en las variables LZ y CAD. Además, dichos autores afirman que una sobrecarga persistente del aparato locomotor no tiene ningún efecto significativo sobre la $V$ de marcha, la LZ o la CAD.

Por otro lado, respecto al análisis de las diferentes fases del ciclo de marcha, se observa que el grupo con sobrepeso reduce significativamente la FO respecto al grupo normopeso lo cual da lugar a un incremento significativo de la FA y en concreto, de la FAB En concordancia con lo anterior, estudio previos ${ }^{10,30}$ concluyen que los sujetos con sobrepeso aumentan de forma significativa su FO respecto a los sujetos sanos, obteniendo unos valores muy similares a los de nuestro estudio.

En estudios previos ${ }^{11,12,14}$ se destaca que un incremento de la FO es un mecanismo clave de ajuste compensatorio durante la locomoción. Estos cambios compensatorios son consecuencia del aumento de la inestabilidad durante la marcha en sujetos obesos debido al sobrepeso, que se acompaña de una menor FO y un incremento en la FA y FAB 9,12.
Como limitaciones encontramos que la muestra está compuesta sólo por hombres jóvenes y activos, siendo también el grupo sobrepeso menos numeroso que el grupo de normopeso. Otra limitación es no incluir un grupo de obesos (IMC >30), para ver diferencias entre sujetos con sobrepeso y obesidad así como la no inclusión de mujeres. Además la configuración de los grupos ha sido en base a IMC teniendo en cuenta las limitaciones de esta medida.

\section{CONCLUSIÓN}

Como conclusión destacar por tanto que los sujetos varones activos con sobrepeso presentan alteraciones en las fases temporales de la locomoción en comparación con los sujetos varones sanos. Estos cambios se producen por el decremento significativo del tiempo de la FO de los sujetos con sobrepeso y el incremento significativo en la FA y FAB.

\section{AGRADECIMIENTO}

Este trabajo es parte del proyecto Promoción de Actividad Física Saludable y Mejora de la Calidad de Vida mediante un Sistema Ergonómico de Evaluación Integral y Prevención de Riesgo (acrónimo: PAQOL) financiado por el Ministerio de Ciencia e Innovación del Gobierno de España, ref: DEP2009-11850. Además, este trabajo ha sido financiado por el Ministerio de Educación, Cultura y Deporte, en la convocatoria de Ayudas del Programa de Formación del Profesorado Universitario (ref. FPU13/00162).

Todos los autores declaran que no hay ningún potencial conflicto de intereses con referencia a este artículo.

CONTRIBUCIÓN DE LOS AUTORES: Cada autor contribuyó individualmente y significativamente al desarrollo del manuscrito. SMC (0000-0002-6363-2967)* y JMHJ (00000003-0053-5884)* contribuyeron en la idea y la escritura del manucrito inicial. EOG (0000-0001-8893-3996)* y VSH (0000-0002-0213-5844)*ayudaron en el procesamiento, análisis y representación de los datos y el contenido. Todos los autores contribuyeron en la escritura, revisión y en la consistencia de los resultados, en la organización de los contenidos, leyeron y aprobaron la versión final del manuscrito.*ORCID (Open Researcher and Contributor ID).

\section{REFERENCIAS}

1. Wearing SC, Hennig EM, Byrne NM, Steele JR, Hills AP. The biomechanics of restricted movement in adult obesity. Obes Rev. 2006;7(1):13-24

2. Lai DT, Begg RK, Palaniswami M. Computational intelligence in gait research: a perspective on current applications and future challenges. IEEE Trans Inf Technol Biomed.. 2009;13(5):687-702.

3. Alguren B, Fridlund B, Cieza A, Sunnerhagen KS, Christensson L. Factors Associated With Health-Related quality of life after stroke: a 1-year prospective cohort study. Neurorehabil Neural Repair. 2012;26(3):266-74.

4. Soh SE, Morris ME, McGinley JL. Determinants of health-related quality of life in Parkinson's disease: a systematic review. Parkinsonism Relat Disord. 2011;17(1):1-9.

5. Paterson K, Hill K, Lythgo N. Stride dynamics, gait variability and prospective falls risk in active community dwelling older women. Gait Posture. 2011;33(2):251-5.

6. Studenski S, Perera S, Patel K, Rosano C, Faulkner K, Inzitari M, et al. Gait speed and survival in older adults. JAMA. 2011;305(1):50-8.

7. Anandacoomarasamy A, Caterson I, Sambrook P, Fransen M, March L. The impact of obesity on the musculoskeletal system. Int J Obes. 2008;32(2):211-22.

8. Rodriguez-Rodriguez E, Lopez-Plaza B, Lopez-Sobaler AM, Ortega RM. Overweight and obesity among spanish adults. Nut Hosp. 2011;26(2):355-63.

9. Hills AP, Hennig EM, Byrne NM, Steele JR. The biomechanics of adiposity-structural and functional limitations of obesity and implications for movement. Obes Rev. 2002;3(1):35-43.

10. Lai PP, Leung AK, Li AN, Zhang M. Three-dimensional gait analysis of obese adults. Clin Biomech. 2008;23(Suppl 1):S2-6.

11. McGraw B, McClenaghan BA, Williams HG, Dickerson J, Ward DS. Gait and postural stability in obese and nonobese prepubertal boys. Arch Phys Med Rehabil. 2000;81(4):484-9.

12. Spyropoulos P, Pisciotta JC, Pavlou KN, Cairns MA, Simon SR. Biomechanical gait analysis in obese men. Arch Phys Med Rehabil. 1991;72(13):1065-70.

13. Wu X, Lockhart TE, Yeoh HT. Effects of obesity on slip-induced fall risks among young male adults. J Biomech. 2012;45(6):1042-7.

14. Hulens M, Vansant G, Claessens AL, Lysens R, Muls E. Predictors of 6-minute walk test results in lean, obese and morbidly obese women. Scand J Med Sci Sports. 2003;13(2):98-105.

15. LaRoche DP, Kralian RJ, Millett ED. Fat mass limits lower-extremity relative strength and maximal walking performance in older women. J Electromyogr Kinesiol. 2011;21(5):754-61.

16. Sartorio A, Proietti M, Marinone PG, Agosti F, Adorni F, Lafortuna CL. Influence of gender, age and BMI on lower limb muscular power output in a large population of obese men and women. Int J Obes Relat
Metab Disord.. 2004;28(1):91-8.

17. Browning RC, Kram R. Energetic cost and preferred speed of walking in obese vs. normal weight women Obes Res. 2005;13(5):891-9.

18. Browning RC, McGowan CP, Kram R. Obesity does not increase external mechanical work per kilogram body mass during walking. J Biomech. 2009;42(14):2273-8.

19. Hoffman MD, Millet GY, Candau RB, Rouillon JD. Evaluation of a theoretical model to quantify the sources of metabolic cost in walking. Am J Phys Med Rehabil. 2004;83(5):353-62.

20. Blaszczyk JW, Plewa M, Cieslinska-Swider J, Bacik B, Zahorska-Markiewicz B, Markiewicz A. Impact of excess body weight on walking at the preferred speed. Acta Neurobiol Exp. 2011;71(4):528-40.

21. Ko SU, Stenholm S, Ferrucci L. Characteristic gait patterns in older adults with obesity-results from the Baltimore Longitudinal Study of Aging. J Biomech. 2010;43(6):1 104-10.

22. WHO. Obesity: preventing and managing the global epidemic. Report of a WHO consultation: World Health Organ Tech Rep Ser. 2000;894:1(12):1--253.

23. Glatthorn JF, Gouge S, Nussbaumer S, Stauffacher S, Impellizzeri FM, Maffiuletti NA. Validity and reliability of optojump photoelectric cells for estimating vertical jump height. J Strength Cond Res. 201 1;25(2):556-60.

24. Malavolti M, Mussi C, Poli M, Fantuzzi AL, Salvioli G, Battistini N, et al. Cross-calibration of eight-polar bioelectrical impedance analysis versus dual-energy $X$-ray absorptiometry for the assessment of total and appendicular body composition in healthy subjects aged 21-82 years. Ann Hum Biol. 2003;30(4):380-91.

25. Sartorio A, Malavolti M, Agosti F, Marinone PG, Caiti O, Battistini N, et al. Body water distribution in severe obesity and its assessment from eight-polar bioelectrical impedance analysis. Eur J Clin Nut. 2005;59(2):155-60.

26. Kressig RW, Beauchet O, European GAITRite Network Group. Guidelines for clinical applications of spatio-temporal gait analysis in older adults. Aging Clin Exp Res. 2006;18(2):174-6.

27. Hollman JH, Childs KB, McNeil ML, Mueller AC, Quilter CM, Youdas JW. Number of strides required for reliable measurements of pace, rhythm and variability parameters of gait during normal and dual task walking in older individuals. Gait Posture. 2010;32(1):23-8.

28. Sánchez Lacuesta JJ, Prat Pastor JM, Hoyos Fuentes JV Viosca Herrero E, Soler Gracia C, Comín Clavijo $\mathrm{M}$, et al. Biomecánica de la marcha humana normal y patológica. Instituto de Biomecánica de Valencia. ed. Valencia.2005:34-97.

29. Runhaar J, Koes BW, Clockaerts S, Bierma-Zeinstra SM. A systematic review on changed biomechanics of lower extremities in obese individuals: a possible role in development of osteoarthritis. Obes Rev 2011;12(12):1071-82

30. Sheehan, KJ, Gormley, J. The influence of excess body mass on adult gait. Clin Biomech. 2013;28(3):337-43. 